\title{
Arterial Stiffness Gradient
}

\author{
Catherine Fortier Mohsen Agharazii \\ CHU de Québec Research Center, L'Hôtel-Dieu de Québec Hospital, and Division of \\ Nephrology, Faculty of Medicine, Université Laval, Québec, Qué., Canada
}

\section{Key Words}

Aortic stiffness - Arterial stiffness gradient - End-organ damage - Medium-sized muscular artery $\cdot$ Mortality

\begin{abstract}
Background: Aortic stiffness is a strong predictor of cardiovascular mortality in various clinical conditions. The aim of this review is to focus on the arterial stiffness gradient, to discuss the integrated role of medium-sized muscular conduit arteries in the regulation of pulsatile pressure and organ perfusion and to provide a rationale for integrating their mechanical properties into risk prediction. Summary: The physiological arterial stiffness gradient results from a higher degree of vascular stiffness as the distance from the heart increases, creating multiple reflective sites and attenuating the pulsatile nature of the forward pressure wave along the arterial tree down to the microcirculation. The stiffness gradient hypothesis simultaneously explains its physiological beneficial effects from both cardiac and peripheral microcirculatory points of view. The loss or reversal of stiffness gradient leads to the transmission of a highly pulsatile pressure wave into the microcirculation. This suggests that a higher degree of stiffness of medium-sized conduit arteries may play a role in protecting the microcirculation from a highly pulsatile forward pressure wave. Using the ratio of carotid-femoral pulse wave velocity (PWV) to carotid-radial PWV, referred to as PWV ratio, a recent study in a dialysis cohort has shown that the PWV ratio is a better predictor of mortality than the classical carotid-femoral PWV. Key Messages: Theoretically, the use of the PWV ratio seems more logical for risk determination than aortic stiffness as it provides a better estimation of the loss of stiffness gradient, which is the unifying hypothesis that explains the impact of aortic stiffness both on the myocardium and on peripheral organs.

(C) 2015 S. Karger AG, Basel
\end{abstract}


Fortier and Agharazii: Arterial Stiffness Gradient

\section{Introduction}

Aortic stiffness, measured by carotid-femoral pulse wave velocity (cf-PWV), is now a well-established predictor of cardiovascular events and mortality in the general population and in pathological conditions such as hypertension, diabetes and chronic kidney disease (CKD) [1-4]. In contrast, the lack of a clear relationship between stiffness of peripheral medium-sized conduit arteries and mortality and cardiovascular outcomes may have overshadowed their physiological importance in the regulation of pulsatile blood pressure for the microcirculation $[5,6]$. The purpose of this review is to underline the importance of mediumsized muscular conduit vessels, to discuss their integrated role in the regulation of pulsatile pressure and organ perfusion and to provide a rationale for integrating their mechanical properties into risk prediction.

\section{Aortic Elasticity and Myocardium}

In physiological conditions, the compliant aorta dampens the pulsatile oscillation of blood pressure and flow during left ventricular cyclic contraction. The elastic aortic recoil during the diastolic phase is essential to maintain a continuous peripheral perfusion. Aortic elasticity is also beneficial for coronary artery perfusion during diastole since the forward pressure waves travel at a reduced speed and return to the proximal aorta in end-systole and diastole [7]. During aging or in pathological conditions, the aorta becomes stiffer and resists accepting the incoming blood flow following left ventricular contraction. As the aorta stiffens, pressure waves travel faster (higher PWV), and reflected waves return earlier to the ascending aorta, increasing the central augmentation index and pulse pressure, which lead to greater cardiac workload. Moreover, since the bulk of reflected waves occur during systole, its impact on maintaining a diastolic blood pressure diminishes, resulting in enhanced diastolic decay. Hence, the impact of aortic stiffness on the myocardium can be explained by an increase in cardiac workload combined with a decrease in myocardial perfusion. This traditional view was forwarded to explain the association between aortic stiffness, hypertension, left ventricular hypertrophy and heart failure. However, our better understanding of the arterial system has evolved into the concept of arterial stiffness gradient so as to explain the impact of aortic stiffness on the heart and peripheral organs such as the brain and the kidneys.

\section{Stiffness Gradient Hypothesis: From Heart to Peripheral Microcirculation}

The distinct function, diameter and structure of arterial segments of the macrocirculation are the foundation of the heterogeneity of the arterial tree. The loss of elastin content and the decrease in vessel diameter as the distance from the heart increases result in a constant increase in stiffness from the aorta to the periphery, which is referred to as stiffness gradient. Therefore, as the forward wave travels along the arterial tree, a part of the energy is returned upstream by reflection sites that are created by the changes in arterial structure, diameter and stiffness [8]. These reflection sites combined with the elastic aorta are therefore beneficial for coronary perfusion pressure without further increase of myocardial workload as explained in the previous section. Figure 1a, reproduced from Briet et al. [9], elegantly demonstrates the physiological stiffness gradient. The forward pressure wave is gradually attenuated throughout its passage along the arterial tree down to the microcirculation, where the pulsatility is minimal. The stiffness gradient hypothesis simultaneously explains its beneficial effects from both a cardiac and a peripheral microcirculatory point of view. 
Fig. 1. Hemodynamic impact of arterial stiffness gradient. a In normal condition, aortic stiffness is lower than that of medium-sized conduit arteries, creating wave reflections and therefore attenuating pulse pressure transmission to the circulation. $\mathbf{b}$ When aortic stiffness increases with minimal changes of stiffness in mediumsized conduit arteries, the forward pressure wave is less attenuated (i.e. there is less reflection), therefore the forward pulse wave is transmitted to the microcirculation stimulating myogenic response and enhanced wave reflection from the microcirculation. This condition could potentially lead to hypoperfusion and organ dysfunction. Reprinted from Briet et al. [9] with permission.

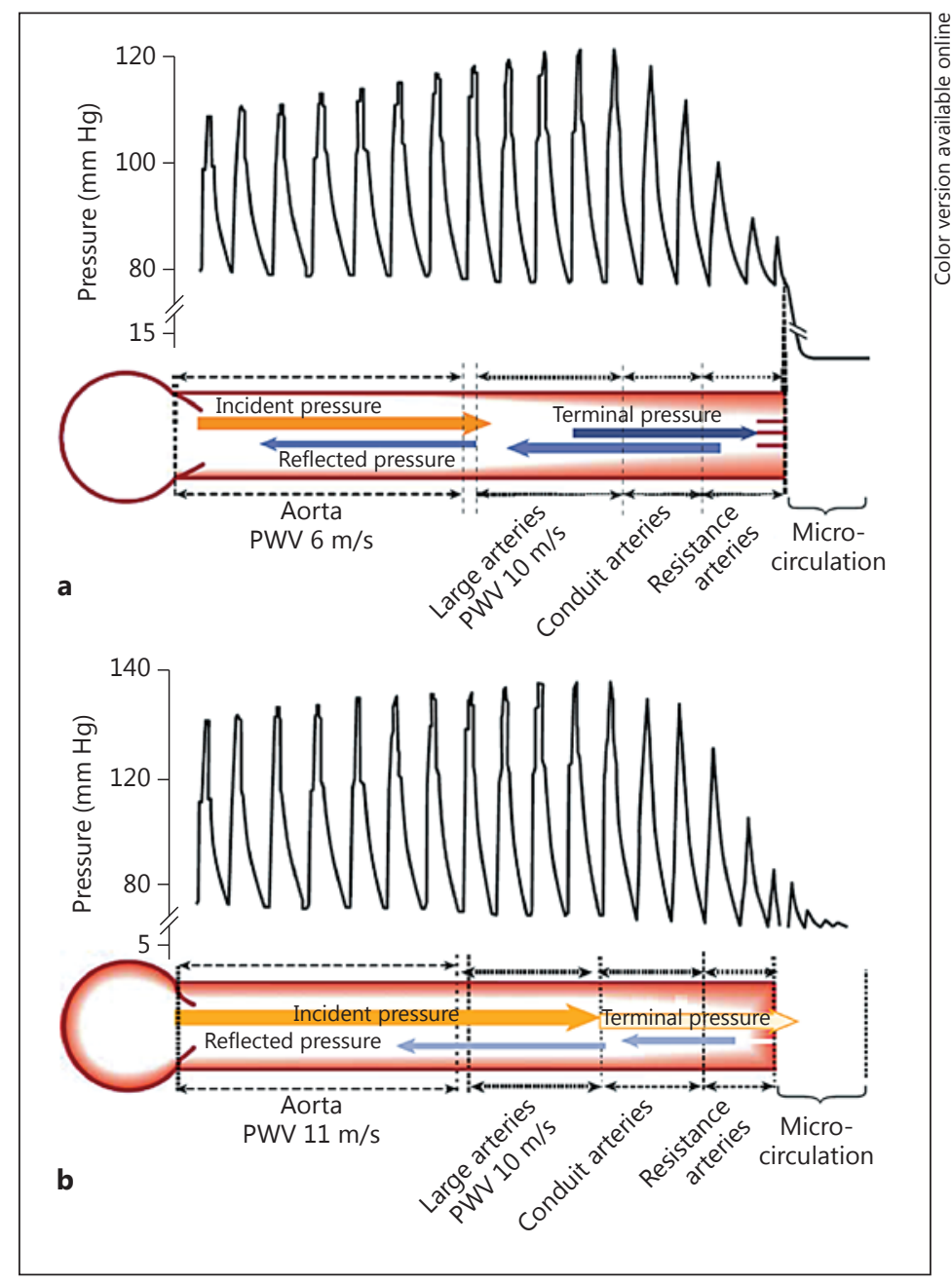

\section{Loss or Reversal of Stiffness Gradient}

Cross-sectional studies have shown that the slope of the relationship between aortic stiffness and age was clearly more pronounced as compared to the slope of the relationship between the stiffness of peripheral muscular arteries and age [10-12]. Furthermore, not only is brachial stiffness associated with a smaller increase with passing years, but it can even slightly decrease after the fifth decade $[13,14]$. This differential vascular stiffness response to aging or pathological conditions will inevitably lead to a reduction, equalization and eventually a reversal of the stiffness gradient. Attenuation or reversal of the stiffness gradient has a significant influence on the central pulse wave profile through a distal shift in major reflection sites, resulting in a dissociation between aortic stiffness and augmentation index $[15,16]$.

The loss of stiffness gradient has also been proposed to be the pathophysiological basis of cardiovascular events and target organ damage that is associated with aortic stiffness (fig. 1b). Indeed, the loss of stiffness gradient is responsible for the transmission of excessive forward pressure into the microcirculation, potentially leading to increased vascular myogenic response, endothelial dysfunction, reduced organ perfusion and ultimately organ dysfunction [17]. Obligate high-flow organs such as the kidneys and the brain may be more susceptible to 
the adverse effects of the loss of stiffness gradient. Indeed, increased pulse pressure, aortic stiffness and pulsatile forward pressure wave have been shown to be associated with increased renal blood flow pulsatility, thus explaining the association between pulse pressure, microalbuminuria and kidney injury [18]. Tarumi et al. [19] showed a negative relationship between aortic stiffness and perfusion of various regions of the brain, which remained statistically significant even after adjustments for age, sex, race, heart rate, blood pressure and cardiovascular medication. In addition, Mitchell et al. [20] reported that aortic stiffness and reduced wave reflection at the carotid-aorta interface were associated with excessive flow pulsatility, which can damage the cerebral microcirculation and results in reduced brain volume with clinical consequences such as increased risk of silent subcortical infarcts and lower scores in various cognitive domains. Finally, Hashimoto and Ito [21] showed that the ratio of cf-PWV/carotid-radial PWV (cr-PWV) remained a significant determinant of reverse/ forward flow ratio in the descending aorta, even after adjustment for potential confounders. Taken together, these studies support the concept that the loss or reversal of arterial stiffness gradient along the arterial tree leads to alterations of blood flow regulation and end-organ damage by the transmission of a higher pulsatile pressure into the microcirculation.

\section{Arterial Stiffness and Stiffness Gradient in CKD}

The mechanisms of aortic stiffness in CKD are complex and still poorly understood. The progression of stiffness may be related to a combination of factors such as premature mechanical fatigue of the elastin lamella due to hypertension, vascular calcification associated with CKD-related mineral disorder, modification of extracellular matrix by advanced glycation end-products (uremic toxins) and endothelial dysfunction [22-24]. In CKD patients in need of hemodialysis, London's group [5] was the first to demonstrate the clinical impact of aortic stiffness on cardiovascular and total mortality. In their population, they showed no clear relationship between brachial and femoral stiffness and mortality.

To determine the rate of progression of aortic stiffness in a longitudinal study, our research team performed repeated measures of aortic stiffness in 109 hemodialysis patients after a mean follow-up of 1.2 years [25]. In this cohort, we showed that cf-PWV increased by an average of $0.84 \mathrm{~m} / \mathrm{s} /$ year after adjusting for changes in mean blood pressure. However, despite the enhanced progression of aortic stiffness, we observed a mean blood pressureadjusted reduction in cr-PWV by $0.66 \mathrm{~m} / \mathrm{s} /$ year. Moreover, a higher baseline aortic stiffness was associated with a greater decline in brachial stiffness. Our work along with that of others $[13,15]$ led us to propose that muscular conduit vessels may adapt to the increased central aortic stiffness by becoming more compliant in order to accept the incoming blood volume. Although the brachial artery does not per se contribute significantly to the total arterial compliance, because of the large number of medium-sized muscular conduit arteries, it is conceivable that these arteries may contribute significantly to the total arterial compliance, especially in the presence of a highly stiff aorta. From the myocardial perspective, a decline in brachial stiffness could be beneficial as it further reduces wave reflections and therefore limits the early arrival of reflecting waves during systole by increasing their transit time. On the other hand, regression of brachial stiffness in conjunction with progression of aortic stiffness may not be desirable as it leads to reversal of the stiffness gradient and a higher forward pressure wave transmission into the microcirculation.

We therefore proposed to study the impact of arterial stiffness gradient by exploring the ratio of cf-PWV/cr-PWV (PWV ratio) on mortality in a cohort of 310 prevalent CKD patients in need of dialysis [26]. After a median follow-up of 29 months, 146 (47\%) deaths occurred. In a Cox proportional hazard model, the risks associated with 1 standardized deviation 
Fig. 2. cf- and cr-PWVs in a dialysis population. a The figure shows increasing cf-PWV (O) and decreasing cr-PWV (O) with increasing age. b Early premature loss of stiffness gradient as evaluated by cf-PWV/cr-PWV (PWV ratio). The results are obtained using data from 310 patients from a prevalent dialysis cohort as reported by Fortier et al. [26], using direct measurements of the distance between two arterial sites. Dots are mean, and bars represent the $95 \%$ CI.

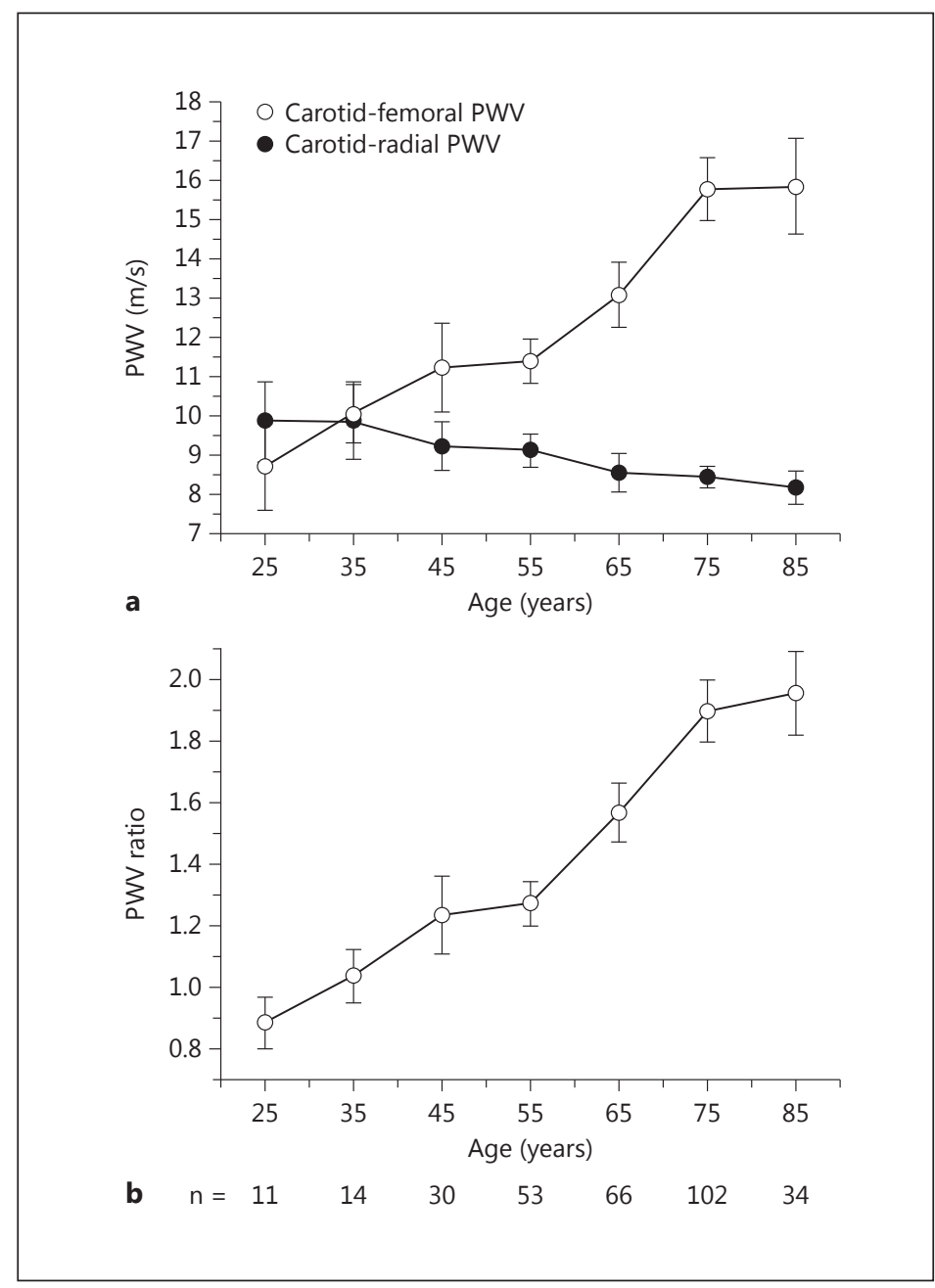

increase in cf-PWV and cr-PWV were 1.29 [95\% confidence interval (CI) 1.11-1.50, p = 0.001] and 0.80 (95\% CI 0.67-0.95, $\mathrm{p}=0.009$ ) suggesting an association between higher cf-PWV and lower cr-PWV with increased risk of mortality. Interestingly, the PWV ratio showed the strongest impact on mortality [hazard ratio (HR) $=1.43,95 \%$ CI 1.24-1.64, p $<0.001$ ]. Moreover, only the PWV ratio remained a significant hemodynamic risk factor after adjustments for confounding factors such as age, dialysis vintage, gender, cardiovascular disease, diabetes, smoking status and weight (HR $=1.23,95 \%$ CI 1.02-1.49). Figure 2 shows the divergent relationships of cf-PWV and cr-PWV with age in our CKD cohort, which is responsible for an increase in PWV ratio.

\section{Lessons, Perspectives and Future Directions}

Theoretically, the use of the PWV ratio is a more logical choice for risk determination than aortic stiffness as it provides a better estimation of the loss of stiffness gradient, which is a unifying hypothesis explaining the impact of aortic stiffness on both myocardium and peripheral organs. At first sight, the PWV ratio might not provide an added value above and beyond that of cf-PWV in the low-risk young-to-middle-age population, since the available evidence shows 
that brachial stiffness remains relatively stable until the fifth decade. Nevertheless, there are still important variations in brachial stiffness within this cohort, and integration of brachial stiffness into risk prediction may provide valuable information on an individual level.

The use of the PWV ratio as a parameter for risk prediction is still at its preliminary stages of development. Further studies are required to assess its capacity to predict cardiovascular events and mortality in dialysis populations as well as in lower-risk populations. One of the limitations of the PWV ratio in our cohort remains the exploration of brachial stiffness as a representative medium-sized muscular conduit vessel. Naturally, additional studies are required to examine the integration of other medium-sized muscular conduit arteries into risk prediction.

Unfortunately, our study was not designed to address the mechanisms of brachial stiffness regression. To our knowledge, no data exist on the longitudinal changes in the structure of the brachial artery, and this will remain an interesting field to explore for years to come. According to the Moens-Korteweg equation $\left(\mathrm{PWV}^{2}=\mathrm{E}_{\text {inc }} \times \mathrm{h} / 2 \mathrm{r} \rho\right)$, a decrease in brachial stiffness will be expected by a reduction in incremental elastic modulus $\left(E_{\text {inc }}\right)$ or a lower wallto-lumen ratio $(\mathrm{h} / 2 \mathrm{r})$. Data from the literature from the prevalent dialysis population show a higher $E_{\text {inc }}$ with a constant wall-to-lumen ratio [27]. However, these studies were conducted in a relatively younger population in which the brachial stiffness was not associated with mortality or cardiovascular events [5]. Therefore, a hypotrophic remodeling is required to explain changes in PWV without affecting $E_{\text {inc. }}$. This is in keeping with the study by Briet et al. [28], which showed a regression of carotid intima media thickness by $22 \mu \mathrm{m} /$ year in a repeated-measure study in a nondialysis CKD cohort. This hypothetical reasoning is further contaminated by the probable violation of the assumptions of isotropic material which ignores the heterogeneous nature of the vascular wall with longitudinal changes in extracellular matrix or functional alterations due to changes in the vascular smooth muscle tone and phenotype. Indeed, vascular smooth muscle cells have been shown to be heterogeneous in terms of mechanical properties and expression/organization of cytoskeleton proteins along the arterial tree. Their mechanical phenotype correlates with the composition of extracellular matrix and can be modulated by cyclic stretching imposed on the cells [29]. Hence, the mechanism behind brachial stiffness regression is not only important from an academic point of view but also from a therapeutic point of view. One can also speculate on the acute and chronic impact of various classes of antihypertensive medication on changes in brachial artery stiffness and PWV ratio.

Finally, in order to overcome the technical difficulties related to the measurement of aortic stiffness, especially in large multicenter studies, there has been a general move towards the development of user friendly devices that evaluate general arterial stiffness, but which also include information about brachial and femoral arteries. The discovery of a regression in brachial stiffness, which may not be beneficial in the presence of increased aortic stiffness, brings into light not only the heterogeneity of the arterial tree in physiological conditions, but also the heterogeneity of response in pathological conditions.

\section{Conclusion}

In this review, we underlined the importance of the stiffness gradient hypothesis and its beneficial physiological impact on both myocardium and peripheral organ perfusion. This hypothesis constitutes the foundation for using the PWV ratio in risk assessment. Thus far, the PWV ratio has been associated with overall mortality in dialysis populations by using the ratio of cf-PWV/cr-PWV. Nevertheless, the validation of the PWV ratio for cardiovascular events and mortality, both in CKD and lower-risk groups of patients, needs further studies. 
Fortier and Agharazii: Arterial Stiffness Gradient

\section{Acknowledgements}

C. Fortier holds a scholarship from Fonds de Recherche du Québec - Santé (FRQ-S). M. Agharazii holds a scholarship from FRQ-S and a research chair in nephrology from the Université Laval.

\section{Disclosure Statement}

The authors have no conflicts of interest to declare.

\section{References}

1 Ben-Shlomo Y, Spears M, Boustred C, May M, Anderson SG, Benjamin EJ, Boutouyrie P, Cameron J, Chen C H, Cruickshank JK, Hwang SJ, Lakatta EG, Laurent S, Maldonado J, Mitchell GF, Najjar SS, Newman AB, Ohishi M, Pannier B, Pereira T, Vasan RS, Shokawa T, Sutton-Tyrell K, Verbeke F, Wang KL, Webb DJ, Willum Hansen T, Zoungas S, McEniery CM, Cockcroft JR, Wilkinson IB: Aortic pulse wave velocity improves cardiovascular event prediction: an individual participant meta-analysis of prospective observational data from 17,635 subjects. J Am Coll Cardiol 2014;63:636-646.

2 Laurent S, Boutouyrie P, Asmar R, Gautier I, Laloux B, Guize L, Ducimetiere P, Benetos A: Aortic stiffness is an independent predictor of all-cause and cardiovascular mortality in hypertensive patients. Hypertension 2001; 37:1236-1241.

3 Blacher J, Guerin AP, Pannier B, Marchais SJ, Safar ME, London GM: Impact of aortic stiffness on survival in end-stage renal disease. Circulation 1999;99:2434-2439.

4 Cruickshank K, Riste L, Anderson SG, Wright JS, Dunn G, Gosling RG: Aortic pulse-wave velocity and its relationship to mortality in diabetes and glucose intolerance: an integrated index of vascular function? Circulation 2002;106:2085-2090.

5 Pannier B, Guerin AP, Marchais SJ, Safar ME, London GM: Stiffness of capacitive and conduit arteries: prognostic significance for end-stage renal disease patients. Hypertension 2005;45:592-596.

6 van Sloten TT, Schram MT, van den Hurk K, Dekker JM, Nijpels G, Henry RM, Stehouwer CD: Local stiffness of the carotid and femoral artery is associated with incident cardiovascular events and all-cause mortality: the Hoorn study. J Am Coll Cardiol 2014;63:1739-1747.

7 O'Rourke MF, Kelly RP: Wave reflection in the systemic circulation and its implications in ventricular function. J Hypertens 1993;11:327-337.

8 Avolio AP, Van Bortel LM, Boutouyrie P, Cockcroft JR, McEniery CM, Protogerou AD, Roman MJ, Safar ME, Segers P, Smulyan H: Role of pulse pressure amplification in arterial hypertension: experts' opinion and review of the data. Hypertension 2009;54:375-383.

9 Briet M, Pierre B, Laurent S, London GM: Arterial stiffness and pulse pressure in CKD and ESRD. Kidney Int 2012;82:388-400.

10 Avolio AP, Chen SG, Wang RP, Zhang CL, Li MF, O'Rourke MF: Effects of aging on changing arterial compliance and left ventricular load in a northern Chinese urban community. Circulation 1983;68:50-58.

11 McEniery CM, Yasmin, Hall IR, Qasem A, Wilkinson IB, Cockcroft JR, Investigators A: Normal vascular aging: differential effects on wave reflection and aortic pulse wave velocity: the Anglo-Cardiff Collaborative Trial (ACCT). J Am Coll Cardiol 2005;46:1753-1760.

12 Kimoto E, Shoji T, Shinohara K, Inaba M, Okuno Y, Miki T, Koyama H, Emoto M, Nishizawa Y: Preferential stiffening of central over peripheral arteries in type 2 diabetes. Diabetes 2003;52:448-452.

13 van der Heijden-Spek JJ, Staessen JA, Fagard RH, Hoeks AP, Boudier HA, van Bortel LM: Effect of age on brachial artery wall properties differs from the aorta and is gender dependent: a population study. Hypertension 2000; 35:637-642.

14 Cameron JD, Bulpitt CJ, Pinto ES, Rajkumar C: The aging of elastic and muscular arteries: a comparison of diabetic and nondiabetic subjects. Diabetes Care 2003;26:2133-2138.

15 Mitchell GF, Parise H, Benjamin EJ, Larson MG, Keyes MJ, Vita JA, Vasan RS, and Levy D: Changes in arterial stiffness and wave reflection with advancing age in healthy men and women: the Framingham Heart Study. Hypertension 2004;43:1239-1245.

16 Vyas M, Izzo JL Jr, Lacourciere Y, Arnold JM, Dunlap ME, Amato JL, Pfeffer MA, Mitchell GF: Augmentation index and central aortic stiffness in middle-aged to elderly individuals. Am J Hypertens 2007;20:642-647.

17 Mitchell GF: Effects of central arterial aging on the structure and function of the peripheral vasculature: implications for end-organ damage. J Appl Physiol (1985) 2008;105:1652-1660.

18 Hashimoto J, Ito S: Central pulse pressure and aortic stiffness determine renal hemodynamics: pathophysiological implication for microalbuminuria in hypertension. Hypertension 2011;58:839-846.

19 Tarumi T, Shah F, Tanaka H, Haley AP: Association between central elastic artery stiffness and cerebral perfusion in deep subcortical gray and white matter. Am J Hypertens 2011;24:1108-1113. 
20 Mitchell GF, van Buchem MA, Sigurdsson S, Gotal JD, Jonsdottir MK, Kjartansson O, Garcia M, Aspelund T, Harris TB, Gudnason V, Launer LJ: Arterial stiffness, pressure and flow pulsatility and brain structure and function: the Age, Gene/Environment Susceptibility - Reykjavik study. Brain 2011;134:3398-3407.

21 Hashimoto J, Ito S: Aortic stiffness determines diastolic blood flow reversal in the descending thoracic aorta: potential implication for retrograde embolic stroke in hypertension. Hypertension 2013;62:542-549.

22 Jono S, McKee MD, Murry CE, Shioi A, Nishizawa Y, Mori K, Morii H, Giachelli CM: Phosphate regulation of vascular smooth muscle cell calcification. Circ Res 2000;87:E10-E17.

23 Kass DA, Shapiro EP, Kawaguchi M, Capriotti AR, Scuteri A, deGroof RC, Lakatta EG: Improved arterial compliance by a novel advanced glycation end-product crosslink breaker. Circulation 2001;104:1464-1470.

24 Demuth K, Blacher J, Guerin AP, Benoit MO, Moatti N, Safar ME, London GM: Endothelin and cardiovascular remodelling in end-stage renal disease. Nephrol Dial Transplant 1998;13:375-383.

25 Utescu MS, Couture V, Mac-Way F, De Serres SA, Marquis K, Lariviere R, Desmeules S, Lebel M, Boutouyrie P, Agharazii M: Determinants of progression of aortic stiffness in hemodialysis patients: a prospective longitudinal study. Hypertension 2013;62:154-160.

26 Fortier C, Mac-Way F, Desmeules S, Marquis K, De Serres SA, Lebel M, Boutouyrie P, Agharazii M: Aorticbrachial stiffness mismatch and mortality in dialysis population. Hypertension 2015;65:378-384.

27 Verbeke FH, Agharazii M, Boutouyrie P, Pannier B, Guerin AP, London GM: Local shear stress and brachial artery functions in end-stage renal disease. J Am Soc Nephrol 2007;18:621-628.

28 Briet M, Collin C, Karras A, Laurent S, Bozec E, Jacquot C, Stengel B, Houillier P, Froissart M, Boutouyrie P; Nephrotest Study Group: Arterial remodeling associates with CKD progression. J Am Soc Nephrol 2011;22: 967-974.

29 Dinardo CL, Venturini G, Zhou EH, Watanabe IS, Campos LC, Dariolli R, da Motta-Leal-Filho JM, Carvalho VM, Cardozo KH, Krieger JE, Alencar AM, Pereira AC: Variation of mechanical properties and quantitative proteomics of VSMC along the arterial tree. Am J Physiol Heart Circ Physiol 2014;306:H505-H516. 